\title{
Critérios de Remissão e Melhora Clínica nas Doenças Reumáticas Pediátricas
}

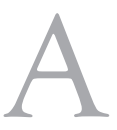
s doenças reumatológicas pediátricas, particularmente a artrite idiopática juvenil/artrite reumatóide juvenil (AIJ/ARJ), o lúpus eritematoso sistêmico juvenil (LESJ) e a dermatomiosite juvenil (DMJ) são raras, com alto impacto físico, mental, emocional, econômico e social nos pacientes e familiares.

Nos últimos 20 anos, duas organizações internacionais merecem destaque na Reumatologia Pediátrica (RP) por condução de estudos colaborativos, multicêntricos e internacionais: Pediatric Rheumatology Collaborative Study Group (PRCSG) e Pediatric Rheumatology International Trials Organization (PRINTO).

O PRCSG foi fundado por Earl Brewer e posteriormente conduzido por Edward H. Giannini e Daniel J. Lovell, com importância na realização de alguns estudos terapêuticos controlados, particularmente na AIJ, nas décadas de 1980 e 1990. O PRINTO foi fundado em 1996 por iniciativa de Alberto Martini e Nicolino Ruperto, inclui 43 países distribuídos mundialmente, com cerca de 170 reumatologistas pediátricos inscritos. Atualmente, os seguintes Estados e serviços de RP brasileiros têm participado de estudos multicêntricos e estão aliados ao PRINTO: São Paulo (UNESP, UNIFESP, FMRP-USP, UNICAMP, Reumatologia da FMUSP e Departamento de Pediatria da FMUSP), Rio de Janeiro (UFRJ-IPPMG, UFRJ-Clínica Médica e UERJ), Paraná (Hospital Pequeno Príncipe), Rio Grande do Sul (UFRGS) e Minas Gerais (UFU-MG).

Nesta edição da Revista Brasileira de Reumatologia, dois importantes artigos de revisão, redigidos por Machado e Ruperto, merecem destaque no universo recente da $\mathrm{RP}^{(1,2)}$. Os artigos incluem critérios de doença inativa e remissão para AIJ/ARJ e critérios de melhora clínica para LESJ e DMJ. Estes foram realizados por membros do PRCSG, PRINTO e Childhood Arthritis and Rheumatology Research Alliance (CARRA).

A AIJ/ARJ é a principal causa de artrite crônica na faixa etária pediátrica. Apesar de recentes estudos em AIJ, tais como: revisão da classificação do $\operatorname{ILAR}^{(3)}$, validação e adaptação para o português brasileiro de instrumentos de qualidade de vida (como CHAQ e CHQ) ${ }^{(4)}$, padronização dos critérios de melhora clínica (ACR Pediátrico 30) ${ }^{(5)}$ e estudos controlados com drogas (etanercepte e metotrexato $)^{(6,7)}$, existiam na literatura médica várias definições de doença inativa e remissão para esta doença. Entre os anos 2000 e 2001, avaliando-se 24 estudos publicados com o termo ARJ, apenas três utilizaram a mesma definição de remissão.

Machado e Ruperto ${ }^{(1)}$ ressaltam os critérios preliminares de doença inativa e remissão clínica (com e sem medicação) propostos recentemente para $\mathrm{AJJ}^{(8)}$. Estes critérios foram desenvolvidos de forma semelhante aos critérios de remissão clínica da artrite reumatóide propostos por Pinals et al. ${ }^{(9)}$. O método Delphi e o Consensus Conference - Nominal Group Techinique foram utilizados para o desenvolvimento destes critérios para as quatro formas de início da AIJ: oligoarticular (persistente ou estendida), poliarticular FR positivo, poliarticular FR negativo e sistêmica. Estes critérios apresentaram validade de construção, abrangência e conteúdo. Por sua vez, ainda estão em desenvolvimento e necessitam validação prospectiva em pacientes reais, assim como precisam ser validados nas outras duas formas da AIJ: artrite psoriática e artrite associada a entesite. Algumas características clínicas da $\mathrm{AIJ}$ não obtiveram consenso entre os especialistas e merecerão validação prospectiva, tais como: rigidez matinal, hepatomegalia e nódulos subcutâneos. A definição de uveíte ativa e inativa também deverá ser realizada.

O LESJ e a DMJ são doenças com manifestações clínicas heterogêneas, cursos imprevisíveis e com alta morbimortalidade. Os estudos terapêuticos com medicamentos avaliando eficácia e segurança nessas doenças incluem pequenas populações, estudos não controlados ou com experiências adaptadas da população adulta. A padronização de critérios de melhora clínica é extremamente importante para a realização de estudos controlados com drogas para LESJ e DMJ ${ }^{(10)}$, assim como foi padronizado e validado na AIJ/ARJ e atualmente conhecido como critério ACR Pediátrico $30^{(5)}$. 
Machado e Ruperto ${ }^{(2)}$ revisam as definições de melhora clínica propostas recentemente para LESJ e DMJ, desenvolvidas por meio de um consenso entre reumatologistas pediátricos. Estas variáveis foram testadas em uma casuística representativa e evidenciaram responsividade e validade discriminativa.

Esses critérios serão ferramentas importantes na reumatologia pediátrica, poderão ser utilizados em futuros

\section{REFERÊNCIAS}

1. Machado C, Ruperto N: Consenso em reumatologia pediátrica. Parte I - Definição dos critérios de doença inativa e remissão em artrite idiopática juvenil/artrite reumatóide juvenil. Rev Bras Reumatol 45: 9-13, 2005.

2. Machado C, Ruperto N: Consenso em reumatologia pediátrica. Parte II - Definição de melhora clínica para o lúpus eritematoso sistêmico e dermatomiosite juvenil. Rev Bras Reumatol 45: 14-19, 2005.

3. Petty RE, Southwood TR, Manners P, et al: International League of Associations for Rheumatology classification of juvenile idiopathic arthritis: second revision, Edmonton, 2001. J Rheumatol 31: 390-2, 2004.

4. Machado CS, Ruperto N, Silva CH, et al: The Brazilian version of the Childhood Health Assessment Questionnaire (CHAQ) and the Child Health Questionnaire (CHQ). Clin Exp Rheumatol 19: S2530, 2001.

5. Giannini EH, Ruperto N, Ravelli A, Lovell DJ, Felson DT, Martini A: Preliminary definition of improvement in juvenile arthritis. Arthritis Rheum 40: 1202-9, 1997. ensaios terapêuticos e metanálises, assim como na prática clínica diária.

Clovis Artur Almeida da Silva Doutor em Medicina pela Faculdade de Medicina da Universidade de São Paulo (FMUSP), São Paulo-SP, Brasil. Responsável pela Unidade de Reumatologia Pediátrica do Departamento de Pediatria da FMUSP

6. Lovell DJ, Giannini EH, Reiff A, et al: Etanercept in children with polyarticular juvenile rheumatoid arthritis. Pediatric Rheumatology Collaborative Study Group. N Engl J Med 342: 763-9, 2000.

7. Ruperto N, Murray KJ, Gerloni V, et al: A randomized trial of parenteral methotrexate comparing an intermediate dose with a higher dose in children with juvenile idiopathic arthritis who failed to respond to standard doses of methotrexate. Arthritis Rheum 50: 2191201, 2004.

8. Wallace CA, Ruperto N, Giannini E: Preliminary criteria for clinical remission for select categories of juvenile idiopathic arthritis. J Rheumatol 31: 2290-4, 2004.

9. Pinals RS, Masi AT, Larsen RA: Preliminary criteria for clinical remission in rheumatoid arthritis. Arthritis Rheum 24: 1308-15, 1981.

10. Ruperto N, Ravelli A, Murray KJ, et al: Preliminary core sets of measures for disease activity and damage assessment in juvenile systemic lupus erythematosus and juvenile dermatomyositis. Rheumatology 42: 1452-9, 2003. 